\title{
Handwritten Digit Recognition Using Various Machine Learning Algorithms and Models
}

\author{
Pranit Patil, Bhupinder Kaur
}

\begin{abstract}
Handwritten digit recognition is a technique or technology for automatically recognizing and detecting handwritten digital data through different Machine Learning models. In this paper we use various Machine Learning algorithms to enhance the productiveness of technique and reduce the complexity using various models. Machine Learning is an application of Artificial Intelligence that learns from previous experience and improves automatically through experience. We illustrate various Machine learning algorithms such as Support Vector Machine, Convolutional Neural Network, Quantum Computing, K-Nearest Neighbor Algorithm, Deep Learning used in Recognition technique.
\end{abstract}

KEYWORDS- Convolutional Neural Network, Support Vector Machine, HandWritten Digit Recognition, Artificial Intelligence, Deep Learning.

\section{INTRODUCTION}

Currently, Handwritten Digit Recognition is a pivotal concern in computer vision. Machine Learning technology makes a machine efficient to perform pattern or text recognition. In fact, the time complexity of current algorithms or models is very high because of contiguous accumulation and incremental development of handwritten digit sample collection of precision of identification [1].

The major concerns of handwritten digit recognition using machine learning models are illustrated as follow [2]:

- Handwriting patterns differ according to the speaker it is normally quite difficult to recognize even humankind handwritten digits as the writing types vary greatly.

- Similarities between handwritten digits, for instance six and four, that look the same based on writing style. Likewise, one and seven may look the same.

\section{Manuscript received July 23, 2020}

Pranit Patil, Student, B.Tech, Department of Computer Science and Engineering, Lovely Professional University, Phagwara, Punjab, India (email: pranitp2222@gmail.com)

Bhupinder Kaur, Assistant Professor, Department of Computer Science and Engineering, Lovely Professional University, Phagwara, Punjab, India
- There is no suitable and effective ideal Machine Learning model for handwritten digit recognition. Different researchers use different methods but there is no ideally suited model for recognizing handwritten digits.[9] In this paper topic is mainly focused on performance and consistency of machine learning models.

- Using defined image recognition patterns, the handwritten digits cannot be identified, since they are specific for various writers.

Machine Learning had mainly three type of learning as follows:

\section{A. Supervised Learning \\ B. Unsupervised Learning \\ C. Reinforcement Learning}

\section{A. Supervised Learning}

Supervised Learning in which model is trained with a labeled dataset. We have two variables one is input and other is output and we have to map from input to the output. Like input(x) and output(y).

$\mathrm{y}=\mathrm{f}(\mathrm{x})$

Example of Supervised Learning Algorithm:

- Naive Bayes

- Random Forest

- Linear Regression

- Support Vector Machine

- K-Nearest Neighbor

- Decision Trees

\section{B. Unsupervised Learning}

Unsupervised Learning in which you need to allow the model to work on unlabeled data, non-classified or categorized. In these we have only input data and to input we don't have output data, i.e. input variable(x) not have output variable.

Example of unsupervised Learning Algorithm:

- K-mean clustering

- Neural Network

- Multivariate Analysis

- Anomaly detection

\section{Reinforcement Learning}

Reinforcement learning is about sequential decision taking. To what output is dependent on current input and next input depends on previous output input.

Example of Reinforcement Learning algorithm:

- Positive 
- Negative

- Markov Decision Process

- Q Learning

\section{LITERATURE SURVEY}

\begin{tabular}{|c|c|c|c|}
\hline Year & Paper Title & Abstract & Analysis \\
\hline IJTP[1] & $\begin{array}{l}\text { Usage of Quantum } \\
\text { K-Nearest Neighbor } \\
\text { Algorithm to improve } \\
\text { handwritten digit } \\
\text { recognition }\end{array}$ & $\begin{array}{l}\text { Study the efficiency of } \\
\text { quantum computing using } \\
\text { Grover Algorithm and K- } \\
\text { Nearest Algorithm }\end{array}$ & $\begin{array}{l}\text { Before this Algorithm time complexity was } \\
12 \mathrm{R} \text { after these algorithm complexity reduce } \\
\text { to me } \mathrm{O}(\mathrm{kM} 2) \text { for accuracy } \\
\text { Algorithm - K- Nearest Neighbor }\end{array}$ \\
\hline ICOASE[2] & $\begin{array}{l}\text { A comparison of three } \\
\text { classification algorithms } \\
\text { for the identification of } \\
\text { handwritten digits }\end{array}$ & $\begin{array}{l}\text { Comparison of three } \\
\text { classification algorithms In } \\
\text { other terms Multilayer } \\
\text { Perceptron (MLP), Naive } \\
\text { Bayes(NB), and K-Star }\end{array}$ & $\begin{array}{l}\text { After Evaluation for all algorithms on } 46 \mathrm{~K} \\
\text { instances with } 10 \text { cross validations for these } \\
\text { K-star get highly accuracy of } 82.36 \% \text { follow by } \\
\text { NB of } 67.04 \% \text { then MLP by } 78.35 \% \text {. } \\
\text { Algorithm: K-Star }\end{array}$ \\
\hline IJECE[3 & $\begin{array}{l}\text { Recognition of } \\
\text { handwritten digits with } \\
\text { classification of decision } \\
\text { tree: A machine learning } \\
\text { method }\end{array}$ & $\begin{array}{l}\text { This paper tested the } \\
\text { standard digital dataset from } \\
\text { kaggle for recognition of } \\
\text { handwritt-en digits using a } \\
\text { machine learning algorithm } \\
\text { decision tree. Accuracy will } \\
\text { from } 0-9 \text { digits }\end{array}$ & $\begin{array}{l}\text { In these model is trained using decision tree } \\
\text { algorithm with a standard dataset consist of } 42 \mathrm{~K} \\
\text { rows and } 720 \text { columns and from this model the } \\
\text { accuracy came to be } 83.4 \% \text {. Accuracy for } 0-9 \\
\text { digits as follows: } \quad 0=83.5 \%, \\
=93.7 \%, \\
\begin{array}{l}4=83.8 \%, \\
7=83.8 \%,\end{array} \\
83.7 \% \\
\text { Algorithm: Decision Tree }\end{array}$ \\
\hline IEEE[4 & $\begin{array}{l}\text { Development of a high } \\
\text { precision handwritten } \\
\text { digit recognition detector } \\
\text { based on a } \\
\text { Convolution-Neural } \\
\text { Network }\end{array}$ & $\begin{array}{l}\text { Determine accuracy and } \\
\text { efficiency using } \\
\text { Convoltion-al neural } \\
\text { network with two layers on } \\
\text { with } 32 \text { images and another } \\
\text { with } 64 \text { images with some } \\
\text { neurons on each layer. }\end{array}$ & $\begin{array}{l}\text { After completion of training with dataset the } \\
\text { accuracy of neural network was found to be } \\
92.6 \% \text { for training set and for test set it was } \\
90.1 \% \text {. Convolutional neural network is much } \\
\text { accurate in-depth learning models and give } \\
\text { excellence performance. } \\
\text { Algorithm: CNN }\end{array}$ \\
\hline MDPI[5 & $\begin{array}{l}\text { Improved Handwritten } \\
\text { Digit Recognition Using } \\
\text { Convolution-al Neural } \\
\text { Networks (CNN) }\end{array}$ & $\begin{array}{l}\text { It optimized various SGD } \\
\text { algorithm to improve the } \\
\text { efficiency of handwritten } \\
\text { digit recognition. } \\
\text { In these paper they explore } \\
\text { the various option with } \\
\text { stride size, kernel, padding } \\
\text { number of layers and } \\
\text { receptive. For testing } \\
\text { MNIST dataset is used. }\end{array}$ & $\begin{array}{l}\text { In these algorithm the learning rate in training } \\
\text { parameter is of } 0.01 \text { and epoch count of maximum } \\
4 \text {. The accuracy was achieved third layer } \\
\text { Convolutional neural network was } 99.76 \% \text { and } \\
\text { for fourth layer of (CNN) we highest accuracy rate } \\
\text { for recognition of } 99.76 \% \text {. } \\
\text { For MINST dataset the model of CNN with three } \\
\text { layers with optimizer provided best accuracy of } \\
99.89 \% \\
\text { Algorithm: Optimized CNN }\end{array}$ \\
\hline IEEE[6 & $\begin{array}{l}\text { Strengthening } \\
\text { Handwritten Digit } \\
\text { Recognition with Two } \\
\text { State Q-Learning }\end{array}$ & $\begin{array}{l}\text { Using two Q state it's } \\
\text { become very simple and } \\
\text { easy to due to it required less } \\
\text { parameter to optimize and } \\
\text { easy to function on MNIST } \\
\text { Digital dataset, USPS } \\
\text { dataset and MATLAB } \\
\text { dataset }\end{array}$ & $\begin{array}{l}\text { Accuracy according to two Q state model as } \\
\text { follows: } \\
\begin{array}{ll}\text { is } 99.0 \% & \text { 1. on MNIST dataset accuracy } \\
99.7 \% & \text { 3. on USPS dataset accuracy is } \\
\text { accuracy is } 100.0 \% & \text {. } \\
\text { Algorithm: Double Q Learning }\end{array}\end{array}$ \\
\hline JILSA[7] & $\begin{array}{l}\text { MCS HOG features and } \\
\text { handwritten digit } \\
\text { recognition system based } \\
\text { on SVM }\end{array}$ & $\begin{array}{l}\text { The Histogram of Directed } \\
\text { Gradient (HOG) with a } \\
\text { Support Vector Machine ( } \\
\text { SVM) algorithm is used in } \\
\text { this Multiple Cell Size } \\
\text { (MCS) to identify digits } \\
\text { from the MNIST digital } \\
\text { dataset. }\end{array}$ & $\begin{array}{l}\text { In experiment the } 10 \text { fold cross and independent } \\
\text { test set is used for validation to determine } \\
\text { accuracy and the accuracy for both was founded to } \\
\text { be } 99.26 \% \text { and } 99.36 \% \text { respectively } \\
\text { Algorithm: SVM }\end{array}$ \\
\hline
\end{tabular}




\section{ALGORITHM SURVEY}

We used various Machine Learning algorithms and various models to get best accuracy by testing and training with various digital datasets.

1. K-Nearest Neighbour is a basic algorithm for classification. It is a kind of supervised learning and mainly focused on pattern recognition and detection for intrusion detection

2. K-Star is also called as instance base classifier. This recognition is done by comparing instances of index of pre recognized or classified sample [2].

3. Decision tree [3] is a kind of supervised algorithm for learning and it is mostly used for a problem like classification and solving problems of recognition. [8]In these model we break problem in data by making decisions like a questions in form of 0 and 1.

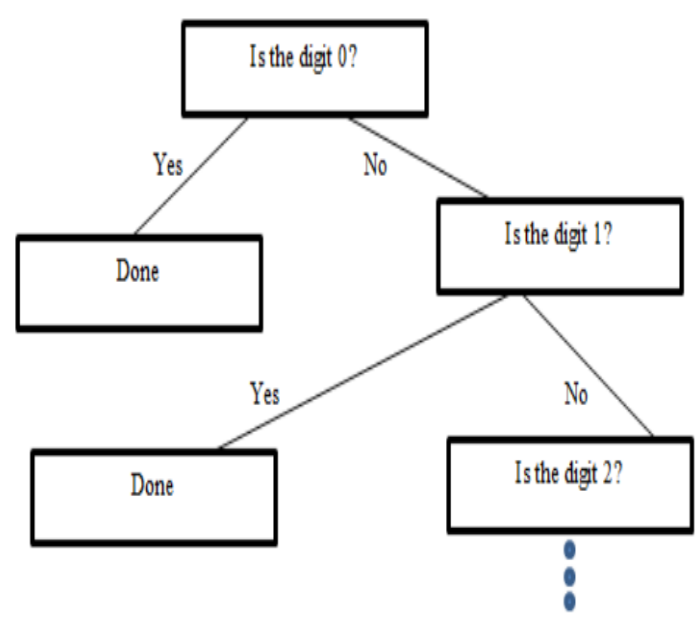

Fig 1: - Decision tree

4. Convolutional Neural Network (CNN) [4] constitutes a type of deep neural network. Some advantages of its are Hidden feature extraction and other is local field and share weights. [5] In deep learning CNN improves the research computer vision and practical improvement and performance. [10],[13]It consists of three layers' Input layer, Hidden layer and classification layer.

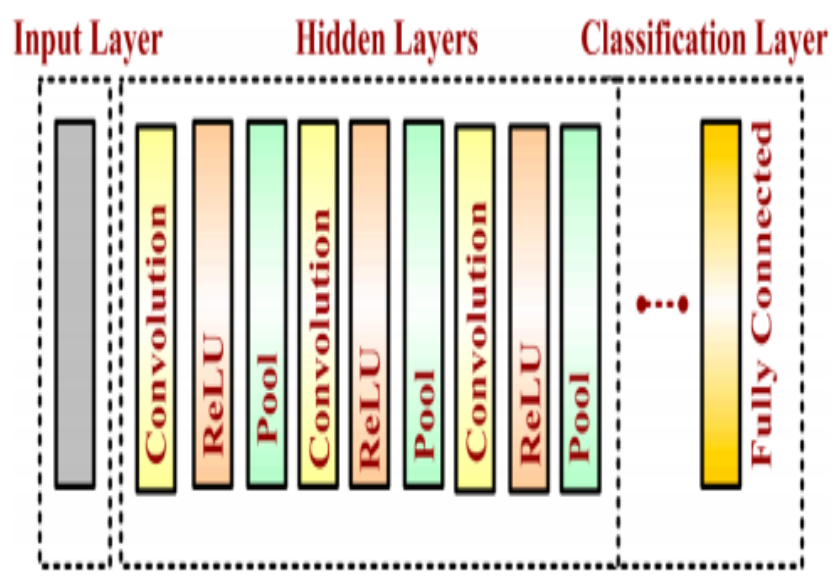

Fig 2: CNN Architecture

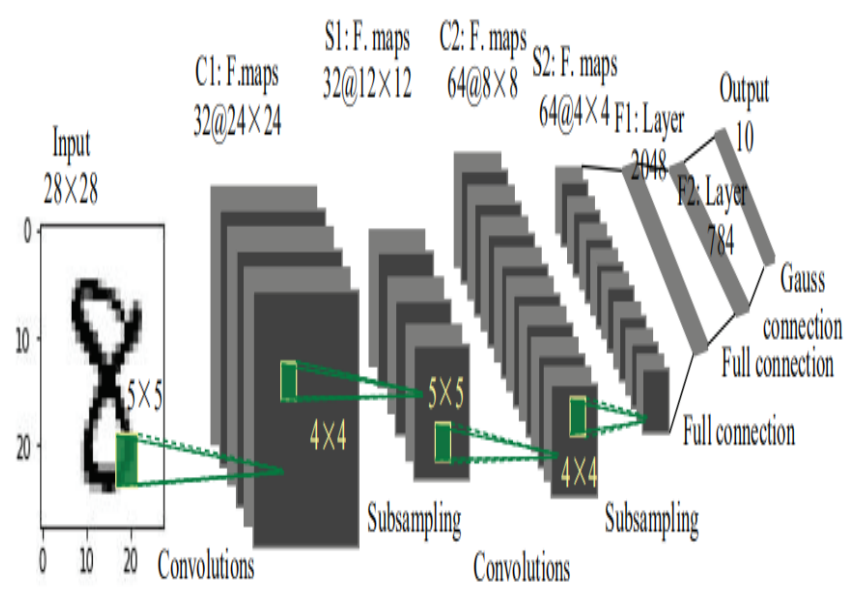

Fig 3: CNN Digit Recognition

5. Double Q Learning [6],[15] used features with huge dimensions along with sates and used in conventional reinforcement network based objects. It is also used for overestimated values. [11]Double Q Learning is faster and efficient then Q Learning.

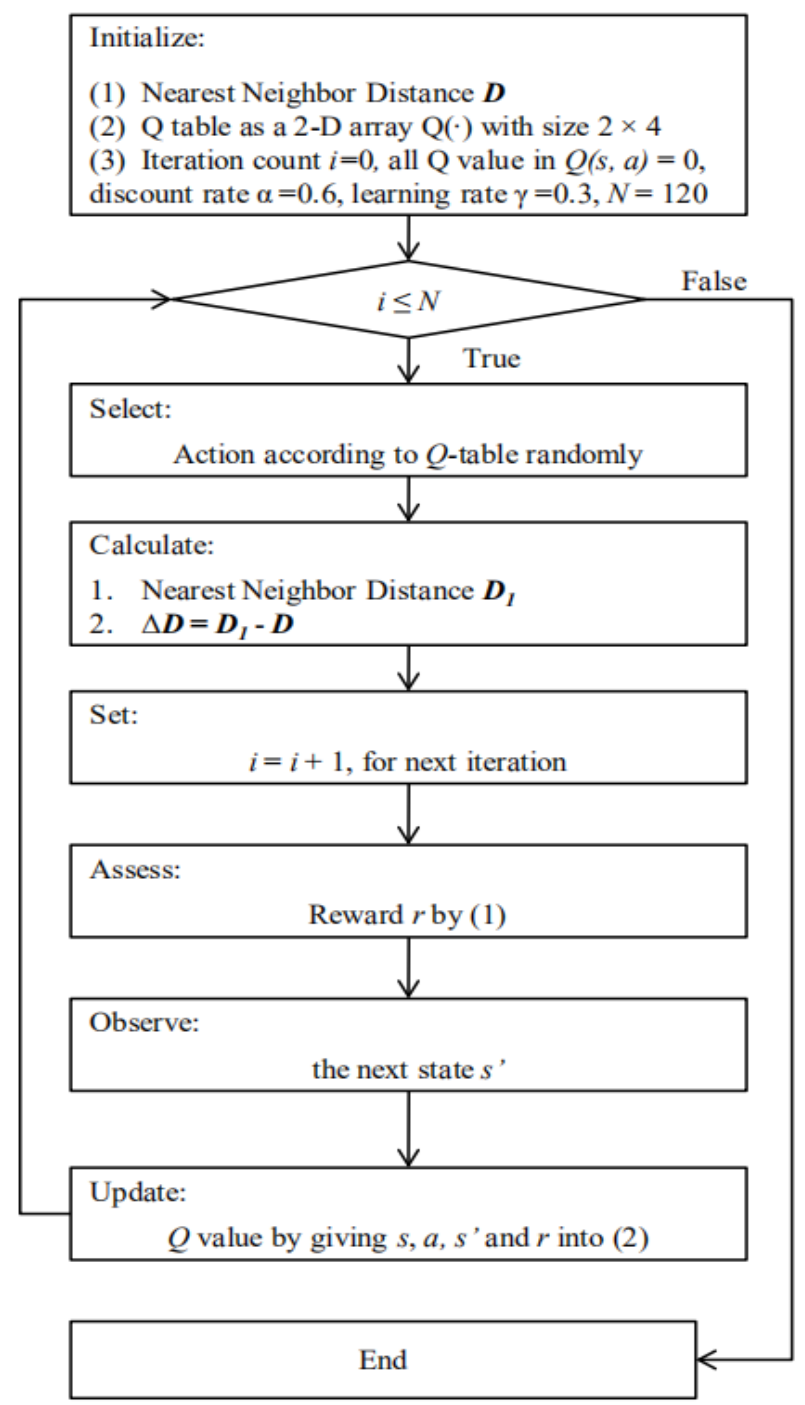

Fig 4: Double Q Learning Recognition algorithm 
6. Support vector Machine (SVM) [7],[14] is a supervised type of machine learning. It is a more accurate classification tool. [12]It results in binary classification or regression challenges. It has capability to handle multiple categorical and continuous variable.

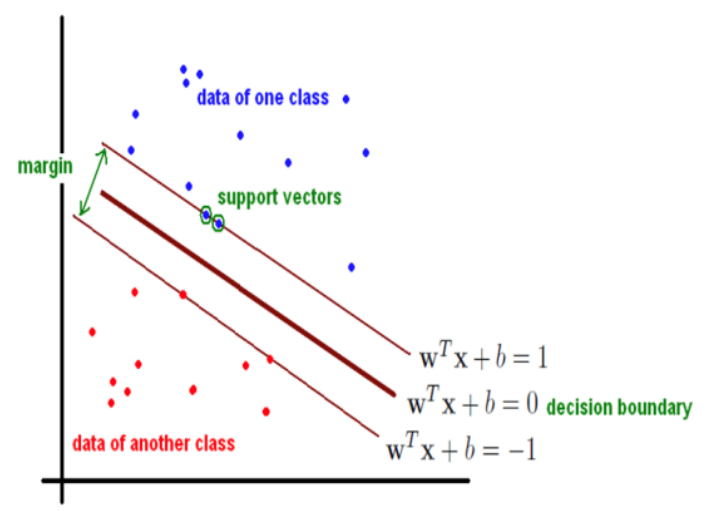

Fig 5: SVM Model

\section{CONCLUSION}

From the survey, it can be achieved that the Machine learning models and algorithms are very efficient to recognize or detect patterns with different writing style. Testing various algorithms gives results in terms of lesser the complexity more will be efficiency and accuracy for any digital data sets. According to survey we have founded that by using Convolutional neural network accuracy increase to the $99.89 \%$ accuracy most among all Similarly, Double Q learning algorithm also given high accuracy but in MATLAB dataset only. SVM also given accuracy of 99.36\%. CNN produce most as it used layered architecture which improve the computer vision and follow a hierarchical model which work on making network and give fully connected layers so neuron get connect to each other and output is processed.

\section{REFERENCES}

[1] Wang, Y., Wang, R., Li, D. et al. Improved Handwritten Digit Recognition using Quantum K-Nearest Neighbor Algorithm. Int J Theor Phys 58, 2331-2340 (2019).

[2] M. B. Abdulrazzaq and J. N. Saeed, "A Comparison of Three Classification Algorithms for Handwritten Digit Recognition," 2019 International Conference on Advanced Science and Engineering (ICOASE), Zakho Duhok, Iraq, 2019, pp. 58-63, doi: 10.1109/ICOASE.2019.8723702.

[3] Assegie, Tsehay \& Nair, Pramod. (2019). Handwritten digits recognition with decision tree classification: a machine learning approach. International Journal of Electrical and Computer Engineering (IJECE). 9. 4446. 10.11591/ijece.v9i5.pp4446-4451. K. Elissa, "Title of paper if known," unpublished.

[4] D. Ge, X. Yao, W. Xiang, X. Wen and E. Liu, "Design of High Accuracy Detector for MNIST Handwritten Digit Recognition Based on Convolutional Neural Network," 2019 12th International Conference on Intelligent Computation Technology and Automation (ICICTA), Xiangtan, China, 2019, pp. 658-662, doi: 10.1109/ICICTA49267.2019.00145.
[5] Al-Wzwazy, Haider. (2016). Handwritten Digit Recognition Using Convolutional Neural Networks. International Journal of Innovative Research in Computer and Communication Engineering. 4.

[6] Hafiz, Abdul \& Bhat, Ghulam. (2020). Reinforcement Learning Based Handwritten Digit Recognition with Two-State Q-Learning. 2007.01193.

[7] Khan, H. (2017) MCS HOG Features and SVM Based Handwritten Digit Recognition System. Journal of Intelligent Learning Systems and Applications, 9, 21-33.

[8] M. Y. W. Teow, "Understanding convolutional neural networks using a minimal model for handwritten digit recognition," 2017 IEEE 2nd International Conference on Automatic Control and Intelligent Systems (I2CACIS), Kota Kinabalu, 2017, pp. 167-172, doi: 10.1109/I2CACIS.2017.8239052.

[9] Alsaafin, A. and Elnagar, A. (2017) A Minimal Subset of Features Using Feature Selection for Handwritten Digit Recognition. Journal of Intelligent Learning Systems and Applications, 9, 55-68.

[10]Wu S., Wei W., Zhang L. (2018) Comparison of Machine Learning Algorithms for Handwritten Digit Recognition. In: Li K., Li W., Chen Z., Liu Y. (eds) Computational Intelligence and Intelligent Systems. ISICA 2017. Communications in Computer and Information Science, vol 874. Springer, Singapore

[11]Jha, G., Cecotti, H. Data augmentation for handwritten digit recognition using generative adversarial networks. Multimed Tools Appl (2020).

[12]Kulkarni, S. R., \& Rajendran, B. (2018). Spiking neural networks for handwritten digit recognition-Supervised learning and network optimization. Neural Networks, 103, 118-127.

[13]Qiao, J., Wang, G., Li, W., \& Chen, M. (2018). An adaptive deep Q-learning strategy for handwritten digit recognition. Neural Networks.

[14]S. Aly and S. Almotairi, "Deep Convolutional Self-Organizing Map Network for Robust Handwritten Digit Recognition," in IEEE Access, vol. 8, pp. 107035-107045, 2020, doi: 10.1109/ACCESS.2020.3000829.

[15]R. Jantayev and Y. Amirgaliyev, "Improved Handwritten Digit Recognition method using Deep Learning Algorithm," 2019 15th International Conference on Electronics, Computer and Computation (ICECCO), Abuja, Nigeria, 2019, pp. 1-4, doi: 10.1109/ICECCO48375.2019.9043235. 